UCB-PTH-05/18

LBNL-57913

\title{
Discretizing Gravity in Warped Spacetime
}

\author{
Lisa Randall $^{a 1}$, Matthew D. Schwartz ${ }^{b 2}$, and Shiyamala Thambyahpillai ${ }^{a 3}$ \\ a Department of Physics, Harvard University \\ Cambridge, MA 02138 \\ ${ }^{b}$ Department of Physics, University of California, Berkeley, \\ and \\ Theoretical Physics Group, Lawrence Berkeley National Laboratory, \\ Berkeley, CA 94720, USA
}

\begin{abstract}
We investigate the discretized version of the compact Randall-Sundrum model. By studying the mass eigenstates of the lattice theory, we demonstrate that for warped space, unlike for flat space, the strong coupling scale does not depend on the IR scale and lattice size. However, strong coupling does prevent us from taking the continuum limit of the lattice theory. Nonetheless, the lattice theory works in the manifestly holographic regime and successfully reproduces the most significant features of the warped theory. It is even in some respects better than the KK theory, which must be carefully regulated to obtain the correct physical results. Because it is easier to construct lattice theories than to find exact solutions to GR, we expect lattice gravity to be a useful tool for exploring field theory in curved space.
\end{abstract}

\footnotetext{
${ }^{1}$ randall@physics.harvard.edu

2 mdschwartz@lbl.gov

${ }^{3}$ thamby@physics.harvard.edu
} 


\section{Introduction}

Warped geometries, such as the background used in Randall-Sundrum model [1,2], have provided many insights into general relativity and holography. However, it is very difficult to find exact solutions to Einstein's equations, so only a handful of warped geometries are known. It would be useful to have a tool for constructing theories that reproduce the features of the warped geometries without having to find and stabilize an appropriate gravitational source. Discrete gravitational dimensions could be such a tool [3-6]. If we work in the effective field theory framework with a cutoff, we may be able to learn a lot about general realtivity without needing exact solutions, as long as the discrete theory can be trusted. Of course, this will not tell us about the energy-momentum tensor to generate such a background, but we can use the discrete model as a tool for investigating stability of the system, the existence of ghosts, and the strong coupling scale, for example. In this paper, we study the the discretization of a single extra dimension in which we impose the exponential warp factor of the Randall-Sundrum model. Although previous work discussed discrete nongravitational extra dimensions for RS [7-12], so far gravity has not been included in the discretized model. We will find that many of the features of the continuum gravitational theory are correctly reproduced, and that some of the problems with flat space lattice gravity are ameliorated.

The discretized model has a number of sites, each of which has a separate fourdimensional metric, and, in the minimal case, has only nearest neighbor links. In the case of flat space, it has been shown that the there is a limit to how small the lattice spacing can be that depends on the overall size of the lattice. This implies that there is no good continuum limit and it is impossible to reproduce 5D gravity all the way up to the five dimensional Planck scale. The absence of the continuum limit in the flat case could be connected to the absence of a simple local holographic description. It seems reasonable that a four-dimensional discretized theory should exist for the warped case, which does have a dual lower-dimensional description.

The flat space strong coupling problems result from two properties of the KaluzaKlein spectrum: there is a very light mode and that mode couples equally over the entire theory space. Both of these properties are the result of the discretized kinetic term, which introduces large mixing between the additional scalars that were originally associated with a single site. The mixing leads to a light highly delocalized mode, whose strong interactions invalidate a low-energy description at a strong coupling scale that lies well below the top of the KK tower. This is a less severe problem in the warped geometry, because the strong warp-factor dependence in the kinetic term keeps the modes localized on only a few sites. The absence of strong mixing means that the localized modes are not lighter than you would naively expect.

However, the warp factor only protects against mixing of modes that are localized on sites further apart than the AdS curvature scale. When the theory is discretized on smaller scales, the theory resembles flat space. Within the AdS curvature scale, 
modes do mix and get delocalized. So although it is possible to reproduce many of the features of the continuum theory with the discretized version, it is still not possible to achieve the true continuum limit. Nevertheless, we find that the dangerous UV/IR problem is absent: the lattice is self-consistent for any number of sites.

\section{Set-up}

We will be concerned with the Poincare patch of $\mathrm{AdS}_{5}$, which is described by the metric

$$
d s^{2}=e^{-2 k y} g_{\mu \nu}(x, y) d x^{\mu} d x^{\nu}+d y^{2} .
$$

As in RS, we impose orbifold boundary conditions at $y=0$ and $y=\pi R$. The $5 \mathrm{D}$ gravitational Lagrangian in this background is

$$
\begin{gathered}
\mathcal{L}=M_{5}^{3} \sqrt{g_{5}}\left(R_{5}-12 k^{2}\right) \\
=M_{5}^{3} \sqrt{g}\left[e^{-2 k y} R_{4}-12 k^{2}+\partial_{y}\left(e^{-2 k y} g_{\mu \nu}\right)\left(g^{\mu \rho} g^{\nu \sigma}-g^{\mu \nu} g^{\rho \sigma}\right) \partial_{y}\left(e^{-2 k y} g_{\rho \sigma}\right)\right] .
\end{gathered}
$$

We are interested in linear fluctuations so we expand $g_{\mu \nu}=\eta_{\mu \nu}+h_{\mu \nu}$. Then, the terms quadratic in $h$, after some integration by parts, are

$$
\mathcal{L}=\frac{1}{4} M_{5}^{3}\left\{e^{-2 k y}\left[h_{\mu \nu} \square h_{\mu \nu}+2 h_{\mu \nu, \alpha}^{2}-2 h_{\mu \nu, \mu} h_{, \mu}+h_{, \mu}^{2}\right]+e^{-4 k y}\left[\left(\partial_{y} h_{\mu \nu}\right)^{2}-\left(\partial_{y} h\right)^{2}\right]\right\} .
$$

To discretize the fifth dimension, we choose points evenly spaced in the $y$-coordinate:

$$
y_{j}=j a, \quad j=0 \cdots N,
$$

where $N$ is the number of lattice sites and $a=R / N$ is the lattice spacing. This is not the only possibility, and discretizing in a different coordinate may result in a drastically different lattice theory. However, $y$ is in some sense the natural choice, since it is a geodesic coordinate for the Poincare patch (unlike, say, the conformal coordinate $z$ ). But more importantly, we can justify using y a posteriori because this discretization will lead to a lattice theory with some of the holographic features of AdS that we expect to see. We replace derivatives in the $y$ direction by differences. For example

$$
e^{-4 k y}\left(\partial_{y} h_{\mu \nu}\right)^{2} \rightarrow \frac{1}{a^{2}} e^{-4 k j a}\left(h_{\mu \nu}^{j+1}-h_{\mu \nu}^{j}\right)^{2} .
$$

This brings out additional ambiguities related to the evaluation of the warping prefactor, and to boundary terms which appear if we integrate by parts before discretizing. However, again, our choices will be justified a posteriori. In any case, if the lattice theory is to be trusted, we should expect that these ambiguities are irrelevant in the continuum limit, which we discuss below. 
Thus we get

$$
\mathcal{L}=\frac{1}{4} M^{2} \sum_{j} e^{-2 k a j} h_{\mu \nu}^{j} \square h_{\mu \nu}^{j}+e^{-4 k a j}\left[\frac{1}{a^{2}}\left(h_{\mu \nu}^{j+1}-h_{\mu \nu}^{j}\right)^{2}-\frac{1}{a^{2}}\left(h^{j+1}-h^{j}\right)^{2}\right],
$$

with $M=\sqrt{M_{5}^{3} a}$ the effective $4 \mathrm{D}$ Planck scale on the sites. Going to canonical normalization

$$
\widehat{h_{j}}=M e^{-k a j} h_{j}
$$

we get a standard lattice action

$$
\mathcal{L}=\frac{1}{4} \sum_{j} \hat{h}_{\mu \nu}^{j} \square \hat{h}_{\mu \nu}^{j}+M_{i j}\left(\hat{h}_{\mu \nu}^{i} \hat{h}_{\mu \nu}^{j}-\hat{h}^{i} \hat{h}^{j}\right)
$$

with mass matrix

$$
M_{i j}=\frac{1}{a^{2}} e^{-2 k a(j-1)}\left[\left(e^{2 k a}+e^{-2 k a}\right) \delta_{i j}-e^{k a} \delta_{i+1, j}-e^{-k a} \delta_{i-1, j}\right] .
$$

Note that this is essentially the same mass matrix as for a gauge boson [7,9-11].

At this point it is helpful to think about the values for $a, k$, and $M$ that we would like to study, which are determined by the continuum limit in which we are interested. As in flat space, we would like to know whether we can have $N \rightarrow \infty$ in such a way that the lattice matches the continuum between any two scales $\Lambda_{\mathrm{UV}}$ and $\Lambda_{\mathrm{IR}}$. In the case of the compact Randall-Sundrum model relevant for the standard model, which has $k \sim M$ and $R \sim 30 M^{-1}$, the limitation $a>M^{-1}$ implies that $N=R / a<30$ in this particular case. This is simply because in RS1 the fifth dimension is only about 30 Planck units wide. Nonetheless, discretization on the scale of $M^{-1}$ would be sufficient in principle to achieve a theory that is valid up to the scale $M$, the same scale as the continuum limit.

In flat space, large $N$ allows you to make a theory that is valid deeper in the infrared. This is also true for the RS model, but only by taking a larger "volume" (that is $R$ ). In this case, $\Lambda_{\mathrm{IR}}$ would be smaller. We will allow $k, N$, and $a$ to be free parameters, with the understanding that $a$ will always be greater than $M^{-1}$ and $N$ will be taken as large as necessary to achieve any desired IR cutoff.

\section{Mass eigenstates}

In this section, we will develop insight into the mass eigenstates of $M_{i j}$. We are working in the regime with $a \approx M^{-1}$ and $k \lesssim M$ so that the space is highly curved. First, we make some quick observations about the mass matrix that will uncover the important qualitative features of the warped lattice. We will afterwards perform a more careful analysis. 


\subsection{Rough, local flat space approximation}

Since $k a<1$, the mass matrix is crudely approximated by

$$
M_{i j} \approx N_{i j} \approx \frac{1}{a^{2}} e^{-2 k a j}\left[2 \delta_{i j}-\delta_{i-1, j}-\delta_{i+1, j}\right]
$$

We have set $e^{k a}=1$ but not $e^{k a j}$, since $j$ may be large. This mass matrix scales with position and is almost diagonal. The only non-diagonal entries are next to the diagonal, because of the nearest-neighbor lattice structure we have assumed. But since the position dependence of the warp factor is exponential, the mass eigenvalues will be geometrically spaced:

$$
m_{n} \approx \frac{1}{a} e^{-k a n}
$$

We confirm this geometric spacing numerically in Figure 1

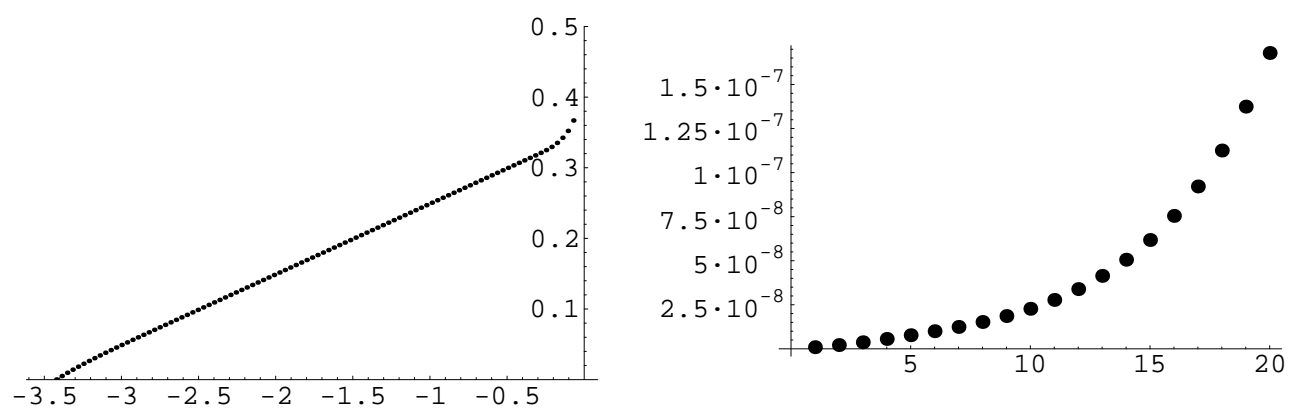

Figure 1: The exact eigenvalues for $N=100$ and $k a=0.1$. On the left is a log plot, showing the geometric spacing. On the right is a close up of the lowest 20 masses, in a non-log plot. Note the linear spacing for the first $1 /(k a)=10$ modes.

This geometric spacing of the masses is very different from the linear spacing of eigenvalues for the KK modes in RS. However, the linear RS spacing is deceptive, since there are only of order $M / k$ relevant at any site. The remaining modes are above the local cutoff, and are in fact localized further in the UV. That is, even though the classical KK tower of RS has an infinite number of linearly spaced modes, no calculation should ever involve more than $M / k$ of them. In fact, the linearized $\mathrm{RS}$ regime does appear, and the geometric spacing is actually quite natural, reproducing the modes localized throughout the bulk with masses determined by the local (warped) AdS scale.

To see this, consider the elements of this matrix around $M_{j_{n} j_{n}}$ for some fixed site $j_{n}$. From Eq. (11),

$$
M_{j_{n}-i, j_{n}-j} \approx \frac{1}{a^{2}} e^{-2 k a j_{n}} e^{2 k a j}\left[2 \delta_{i j}-\delta_{i-1, j}-\delta_{i+1, j}\right] .
$$


For $j<(k a)^{-1}$ the $e^{2 k a j}$ term is approximately 1 . Then in this region the mass matrix looks like flat space. So the eigenstates $H^{(j)}$ (for this $j_{n}$ ) are roughly

$$
H_{i}^{(j)} \sim \sin (\pi k a i j), \quad j_{n}-\frac{1}{k a}<i<j_{n}+\frac{1}{k a}
$$

with eigenvalues

$$
m_{n} \approx j\left(k e^{-k a j_{n}}\right) .
$$

For example, if we look at the lightest modes, with $j_{n}=N$ they are linearly spaced just as in RS. This linear spacing breaks down around $j \sim 1 /(k a) \sim M / k$. This is exactly what we expect because the $M / k$ mode has a mass of the local Planck scale. The linear spacing for the low modes is evident in Figure (11).

In the approximation (13), the prefactor $e^{-2 k a j_{n}}$ implies that there will be little mixing between modes from an expansion around different sites $j_{1}$ and $j_{2}$ if $j_{1}$ $j_{2}>1 /(k a)$. The linear spacing is reconciled with the geometric spacing which is transparent in the exponential prefactor, because the linear spacing appears only when the exponential is roughly constant. We will see that the lack of mixing between modes localized more than of order $k^{-1}$ apart is the reason for the larger regime of validity of the warped discretization, and allows $N$ and $R$ to be taken as large as desired.

The interesting thing about this way of approximating the modes is that it applies for modes centered around any site $j_{n}$. Of course, this is not surprising because of the conformal scaling of RS as we move from the IR to the UV. But it is remarkable that we can get information about a dynamical feature of $\mathrm{RS}$, that the relevant modes change with energy, from a fixed lattice. Indeed, the modes near a site $j_{1}$ and those near a site $j_{2}$ decouple, if $j_{1}-j_{2}>1 /(k a)$. This is very different from what happens to the KK modes $\chi_{n}(y)$ of the continuum RS. If we take two KK modes with masses matching the lattice modes, we find that they do have significant overlap in even if their masses are very different. Of course, the KK picture is not valid at all energies, so we should never be considering two widely spaced modes at the same time. On the lattice we can just use all the modes, and the widely spaced ones naturally decouple from each other. Although to use this rough flat-space approximation, we have to concentrate on a single site or a single mode, we will see that with a more careful analysis of the lattice eigenstates, the same qualitative features still hold.

\subsection{Improved solutions}

Even though this flat space approximation tells us most of what we want to know about the lattice theory, it is instructive to have a representation of the mass eigenstates in the region away from where they have most of their support. This both justifies our approximation, and will be used in the strong coupling calculations below. 
We want to develop a better understanding of the mass eigenstates of the lattice theory. We are looking for the eigenstates of

$$
M_{i j}=\frac{1}{a^{2}} e^{-2 k a(j-1)}\left[\left(e^{2 k a}+e^{-2 k a}\right) \delta_{i j}-e^{k a} \delta_{i+1, j}-e^{-k a} \delta_{i-1, j}\right],
$$

for $k a \lesssim 1$. Let us define a matrix $\eta$ which goes from position eigenstates to mass eigenstates

$$
h_{j}=\eta_{j}^{n} H_{n}
$$

We have already observed from the flat space approximation, that $\eta_{j}^{n} \sim \sin (n j)$ for $\left|j-j_{n}\right|<1 /(k a)$. Now we will confirm this and also approximate the $\eta_{j}^{n}$ in other regions.

One approach is to use the bulk Kaluza-Klein modes to guide the search for the $H_{n}$. The KK equation following from the Lagrangian (4), with $h(x, y)=\sum H_{n}(x) \chi_{n}(y)$, is

$$
\chi^{\prime \prime}(y)-4 k \chi^{\prime}(y)+m_{n}^{2} e^{2 k y} \chi(y)=0 .
$$

It is not hard to see that the equation (18) maps directly onto the eigenvalue equation for $M_{i j}$. The small parameter $k a$ is the step size for the dimensionless continuum variable $\hat{y}=k y$. Then, expanding the derivatives, (18) becomes

$\frac{1}{(k a)^{2}}[\chi(\hat{y}+k a)-2 \chi(\hat{y})+\chi(\hat{y}-k a)]-\frac{4 k}{(k a)} \frac{1}{2}[\chi(\hat{y}+k a)-\chi(\hat{y}-k a)]+m_{n}^{2} e^{2 \hat{y}} \chi(\hat{y})=0$.

For the first derivative, we have taken two steps to symmetrize with respect to \pm .

On the other hand, we can invert (17)

$$
H_{n}=\eta_{n}^{j} e^{-2 k a j} h_{j}
$$

We have used the fact that $H_{n}$ and $\hat{h}_{j}=e^{-k a j} h_{j}$ are canonically normalized, so $\eta_{n}^{j} e^{-k a j}$ is unitary. Then, hitting $H_{n}$ with $M_{i j}$, and projecting out the $h_{j}$ component we find

$$
\frac{1}{a^{2}} e^{-2 k a(j-1)}\left[\left(e^{2 k a}+e^{-2 k a}\right) \eta_{j}^{n}-e^{-2 k a} \eta_{j+1}^{n}-e^{2 k a} \eta_{j-1}^{n}\right]-m_{n}^{2} \eta_{j}^{n}=0 .
$$

For small $k a$ this becomes

$$
\frac{1}{a^{2}}\left[\eta_{j+1}^{n}-2 \eta_{j}^{n}+\eta_{j-1}^{n}-2 k a\left(\eta_{j+1}^{n}-\eta_{j-1}^{n}\right)\right]+e^{2 k a(j-1)} m_{n}^{2} \eta_{j}^{n}=0,
$$

which is the same as (19) for $\eta_{j}^{n}=\chi_{n}(k a j)$.

In the continuum, the solutions to (18) are KK modes:

$$
\chi_{n}(y)=e^{2 k y} \mathcal{J}_{2}\left(\frac{m_{n}}{k} e^{k y}\right) .
$$


The corresponding discrete modes are

$$
\eta_{j}^{n}=e^{2 k a j} \mathcal{J}_{2}\left(\frac{m_{n}}{k} e^{k a j}\right) .
$$

We cannot really manipulate these discrete Bessel functions, but we do expect them to satisfy approximately the same relations as the continuum Bessel functions. For example, the relation

$$
\chi_{n}^{\prime}(y)=e^{3 k a j} \frac{m_{n}}{k} \mathcal{J}_{1}\left(\frac{m_{n}}{k} e^{k a j}\right)
$$

implies

$$
\eta_{j+1}^{n}-\eta_{j}^{n} \approx k a \frac{m_{n}}{k} e^{3 k a j} \mathcal{J}_{1}\left(\frac{m_{n}}{k} e^{k a j}\right) .
$$

Then it is rather trivial to show the modes are eigenstates - they must be because the equations match up.

But note that matching the continuum solutions onto the lattice works because we are using only the first term in the Taylor series, in going from (18) to (19). This is, in fact, all we can do, because we have only included nearest-neighbor links in the discretization. And that means that the solution will break down when the first derivative is no longer a good approximation, i.e. when

$$
1 \approx k a \mathcal{J}^{\prime}\left(\frac{m_{n}}{k} e^{k y}\right) \approx m_{n} a e^{k a j} .
$$

So these solutions should be good until $m_{n} e^{k a j} \approx 1 / a$. In fact, the exact eigenstates of this matrix really look like the continuum KK Bessel functions only in this limited regime. The lattice does not just take a selection of the KK modes, but presents a fundamentally different structure. However, this structure actually reproduces the correct physics, as the heavier KK modes in the continuum theory would be cutoff by the local strong coupling scale.

The Bessel solutions apply for small $j<j_{n}$. That is, they approximate the light KK modes at positions corresponding to energies above their mass. The Bessel functions peak and are localized when

$$
m_{n} e^{k a j_{n}}=k,
$$

which defines $j_{n}$. So, the Bessel approximation is good through the localization region. We also see that the width of the peak is determined by the width of the exponential, that is $\Delta j \approx 1 /(k a)$, so the region where the Bessel function peaks is the same as the region where the flat space approximation applies.

In the flat space region, we can take

$$
\eta_{j}^{n}=e^{2 k a j} \sin \left(\frac{\pi}{3} j\right) \sim e^{2 k a j_{n}} \sin (j),
$$

which satisfies the small $k a$ equation, following from (22):

$$
2 \eta_{j}^{n}-\eta_{j+1}^{n}-\eta_{j-1}^{n}=e^{2 k a j}\left(a m_{n}\right)^{2} \eta_{j}^{n},
$$


with the eigenvalues $m_{n} \sim(1 / a) e^{-k a n}$.

These are not quite the same flat space solutions as the ones derived in the previous subsection from looking at the modes around a particular site. The difference is that in the rough approximation, the heavier modes have higher frequency, because they are the excited states of a box around a particular site. We have seen here that the actual eigenstates all have the same frequency because each one is effectively the zero mode of a different box, centered around the site where the mode is localized. Nonetheless, the important point is that modes of both approximations have the same qualitative features: oscillating behavior with roughly constant amplitude over around $1 /(k a)$ sites.

Now we have solutions for small $j$, in the Bessel regime, and for $j \sim j_{n}$, in the flat space regime. For large $j$, so that $m_{n} a e^{k a j} \gg 1$ the solutions are

$$
\eta_{j}^{n} \approx(-1)^{j} e^{-k a j^{2}} e^{-k a j}\left(m_{n} a\right)^{-2 j} .
$$

We can check

$$
\begin{gathered}
\eta_{j+1}^{n}=-e^{-2 k a j} e^{-2 k a}\left(m_{n} a\right)^{-2} \eta_{j}^{n} \\
\eta_{j-1}^{n}=-e^{2 k a j}\left(m_{n} a\right)^{2} \eta_{j}^{n} .
\end{gathered}
$$

So in this regime $\eta_{j-1}^{n} \gg \eta_{j}^{n} \gg \eta_{j+1}^{n}$ and thus (21) reduces to

$$
-\frac{1}{a^{2}} e^{-2 k a j} \eta_{j-1}^{n}-m_{n}^{2} \eta_{j}^{n}=0,
$$

which is satisfied by our ansatz.

In summary, the solutions for the eigenstates are (up to normalization)

$$
\eta_{j}^{n} \propto\left\{\begin{array}{ll}
e^{2 k a j} \mathcal{J}_{2}\left(\frac{m_{n}}{k} e^{k a j}\right), & j<j_{n} \\
\sin (j), & j \sim j_{n} \\
(-1)^{j} e^{-k a j^{2}} e^{-k a j}\left(m_{n} a\right)^{-2 j}, & j>j_{n}
\end{array} .\right.
$$

To align and normalize the solutions, note that the Bessel solution is exponentially growing

$$
e^{2 k a j} \mathcal{J}_{2}\left(\frac{m_{n}}{k} e^{k a j}\right) \approx\left(\frac{m_{n}}{k}\right)^{2} e^{4 k a j} .
$$

And in the third, large $j$, regime, $\eta_{j}^{n}$ dies as $\exp \left(-k a j^{2}\right)$. So in both of these regimes, the modes have most of their support towards the middle. This means that the normalization is determined almost entirely from the flat space approximation.

Thus we are led back to our original guess. We only need the flat space approximation. If we are looking near a site $j_{n}$ associated with a mode $n$ then

$$
\eta_{j}^{m} \approx\left\{\begin{array}{ll}
\sqrt{k a} \sin (j), & \left|j-j_{n}\right|<1 /(k a) \\
0, & \left|j-j_{n}\right|>1 /(k a)
\end{array} .\right.
$$

Some exact eigenvectors are plotted in Figure2, and the approximation (35) is shown in Figure 3 . 


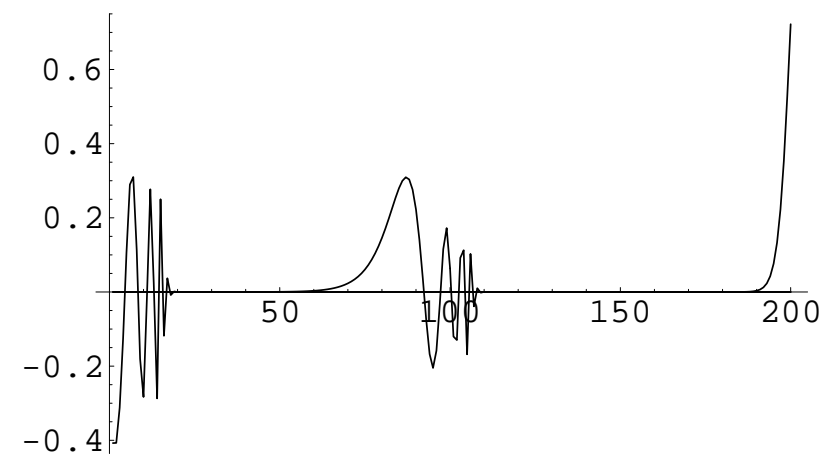

Figure 2: Exact eigenvectors of the mass matrix as a function of site. The numerical solutions for the 10th, 100th and $N$ th eigenstates with $N=200$ and $1 /(k a)=10$ are shown. Heavier modes are on the left. The $N$ th mode is the lightest, and localized on the $N$ th, IR, site.

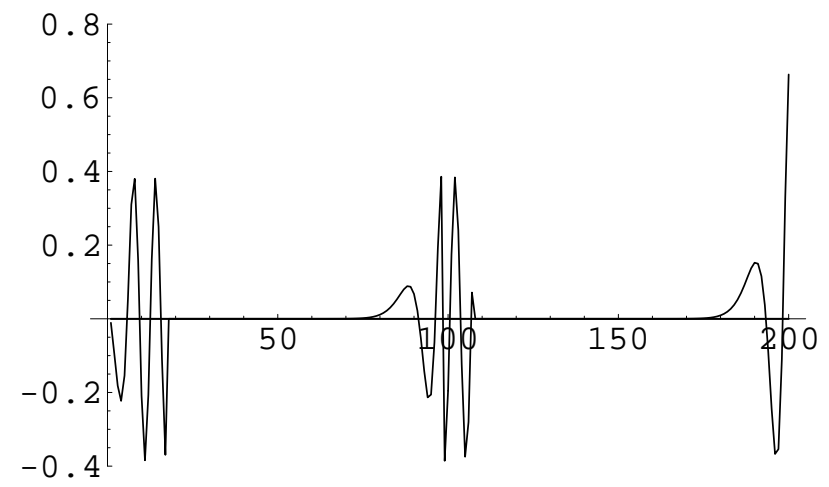

Figure 3: Approximate solutions, with the same parameters as in Figure 2 


\section{$4 \quad$ Strong coupling}

Now that we understand the eigenstates of the lattice theory, we can ask whether there is any limit in which the lattice is a good approximation to the continuum. First, let us recall some difficulties that are encountered in flat space $[5,6]$.

\subsection{Flat space}

To trust the predictions of the lattice theory, it is essential to know the scale at which it becomes nonperturbative and the effective theory breaks down. In a gravitational system, as in a gauge theory, the easiest way to find this scale is to introduce Goldstone bosons restoring a non-linear symmetry, a la CCWZ [13]. This procedure is explained in detail in [4], and we will only briefly review it here. For a massless graviton, this means restoring general coordinate invariance with vector $\left(A_{\alpha}\right)$ and scalar $(\phi)$ Goldstones:

$$
\begin{gathered}
h_{\mu \nu}=g_{\mu \nu}-\eta_{\mu \nu} \rightarrow g_{\mu \nu}-\partial_{\mu} Y^{\alpha} \partial_{\nu} Y^{\beta} \eta_{\alpha \beta} \\
Y_{\alpha} \equiv A_{\alpha}+\phi_{, \alpha} .
\end{gathered}
$$

Then the Fierz-Pauli mass term generates a kinetic term for the scalar

$$
h_{\mu \nu}^{2}-h^{2} \rightarrow h_{\mu \nu}^{2}-h^{2}+\phi_{, \mu, \nu}^{2}-(\square \phi)^{2}+2 \phi_{, \mu, \nu}\left(h_{\mu \nu}-\eta_{\mu \nu} h\right)+(\square \phi)^{3}+\cdots .
$$

The two four-derivative terms for $\phi$ cancel after integration by parts, leaving a proper two-derivative kinetic term for $\phi$ after the $h, \phi$ kinetic matrix is diagonalized. The $\phi$ self-interactions are the strongest and indicate where the theory breaks down. The scales are

$$
\mathcal{L}=M_{P}^{2} h \square h+M_{P}^{2} m^{2} h^{2}+M_{P}^{2} m^{2} \phi \square h+M_{P}^{2} m^{2}(\square \phi)^{3} .
$$

So the canonically normalized fields are $h^{c}=M_{P} h$ and $\phi^{c}=M_{P} m^{2} \phi$ leading to

$$
\mathcal{L}=h^{c} \square h^{c}+\phi^{c} \square h^{c}+\left(M_{P} m^{4}\right)^{-1}(\square \phi)^{3},
$$

from which we read that the cutoff is $\Lambda=\Lambda_{5} \equiv\left(M_{P} m^{4}\right)^{1 / 5}$.

On the flat space lattice, there are many massive gravitons. So we introduce many Goldstones via $\Delta h_{j} \rightarrow \Delta h_{j}+\square \phi_{j}+\cdots$. Then

$$
\mathcal{L}=\sum_{j} M^{2} h_{\mu \nu}^{j} \square h_{\mu \nu}^{j}+\frac{M^{2}}{a^{2}}\left[\left(h_{\mu \nu}^{j+1}-h_{\mu \nu}^{j}\right)^{2}+\left(h_{\mu \nu}^{j+1}-h_{\mu \nu}^{j}\right) \square \phi^{j}+\left(\square \phi^{j}\right)^{3}\right] .
$$

Barring any unforeseen cancellations the strong coupling scale is determined by the interactions of the lightest mode. On the lattice, the effective higher-dimensional 
modes (the KK modes) get contributions from all the site modes, so there is a corresponding $1 / N$ suppression of the interactions. For example, with $h_{j}=\exp (2 \pi i j n / N) H_{n}$

$$
\sum_{j} M^{2} h_{\mu \nu}^{j} \square h_{\mu \nu}^{j} \rightarrow \sum_{n} N M^{2} H_{\mu \nu}^{n} \square H_{\mu \nu}^{n},
$$

so the Planck scale associated with the sites is lower by a factor of $\sqrt{N}$ than the Planck scale of the modes. For the gravity case, there is an additional $N$-dependence for the scalar longitudinal modes because they get their kinetic term from mixing with differences. Then,

$$
\sum_{j} \frac{M^{2}}{a^{2}}\left(h_{\mu \nu}^{j+1}-h_{\mu \nu}^{j}\right) \square \phi^{j} \rightarrow \sum_{n} N \frac{M^{2}}{a^{2}} \frac{1}{N} H \square \Phi .
$$

Thus with $H^{c}=\sqrt{N} M H$ we get $\Phi^{c}=M /\left(\sqrt{N} a^{2}\right) \Phi$. This means that the strongest interaction, from the lightest mode, is

$$
\sum_{j} \frac{M^{2}}{a^{2}}\left(\square \phi^{j}\right)^{3} \rightarrow N \frac{N^{3 / 2} a^{4}}{M}\left(\square \Phi_{1}^{c}\right)^{3}=\frac{1}{N M_{P} m_{1}^{4}}\left(\square \Phi_{1}^{c}\right)^{3},
$$

where $m_{1}=1 / N a$ and $M_{P}=\sqrt{N} M$. Thus the strong interaction for the flat space lattice is at a scale

$$
\Lambda_{\text {flat }}=\left(M N^{-5 / 2} a^{-4}\right)^{1 / 5}=\left(N M_{P} m_{1}^{4}\right)^{1 / 5} .
$$

Since this $M_{P}$ is the low energy Planck scale, and $m_{1}$ is the physical mass, we can see that this scale is higher by a factor of $N^{1 / 5}$ than that of a single massive graviton.

The fact that the strong coupling scale is heavier than the mass of the light mode tells us that this is a good effective theory for the light mode. But for a real lattice description of flat space, this must be a good effective theory all the way up to the 5D Planck scale, $M_{5}^{3}=M_{P}^{2} R^{-1}$. Since $R=m_{1}^{-1}$ we can write the cutoff as $\Lambda_{\text {flat }}=\left(M_{5}^{3} R^{-5} a^{-2}\right)^{1 / 10}$. Then for $\Lambda_{\text {flat }}>M_{5}$ we would need $M_{5} a<\left(R M_{5}\right)^{-5 / 2}$. But since $R M_{5} \gg 1$ this means that $a \ll M_{5}^{-1}$ - the lattice spacing has to be much smaller than the Planck length. But this does not make sense. For the lattice to cut off the divergences from gravity, we must take $a>M_{5}^{-1}$.

Another way to understand the difficulty is to observe that with the lattice spacing at its limit, $a=M_{5}^{-1}$, we get $\Lambda_{\text {flat }}=\sqrt{M_{5} / R} \ll M_{5}$. So there will be new effects at distances much much larger than the Planck length. These non-local effects are most apparent if we work directly in the continuum limit

$$
a^{-2}\left(\Delta h_{j}+\square \phi_{j}+\left(\square \phi_{j}\right)^{2}\right)^{2} \rightarrow\left(\partial_{y} h\right)^{2}+a^{-1}\left(\partial_{y} \phi\right)(\square h)+a^{-2}(\square \phi)^{3}+\cdots
$$

An integration by parts has been performed on the middle term. This shows that $\psi=\partial_{y} \phi$ is the propagating field, while $\phi$ without derivatives is producing the strong 
interactions. In terms of $\psi$ the Lagrangian contains

$$
\mathcal{L}=M_{5}^{3} R h \square h+M_{5}^{3} R h \square \psi+M_{5}^{3} R a\left(\frac{\square}{\partial_{y}} \psi\right)^{3}+\cdots .
$$

The long wavelength modes with $\partial_{y} \sim R^{-1}$ interact at the scale $\Lambda_{\text {flat }}=\left(M_{5}^{3} R^{-5} a^{-2}\right)^{1 / 10}$ we derived above. But we can also now see that the strong interactions are really non-local in the extra dimension. Formally, as $a \rightarrow 0$ (or $N \rightarrow \infty$ ), the strong coupling problem disappears, as it must if this lattice is to classically reproduce the continuum. However, $\Lambda$ does not grow with $N$ fast enough to ensure that the effective theory is consistent.

\subsection{Warped space}

Now let us see how these observations change in the warped background. We introduce Goldstones into the warped space Lagrangian in the usual way

$$
\mathcal{L}=\sum_{j} M^{2} e^{-2 k a j} h_{\mu \nu}^{j} \square h_{\mu \nu}^{j}+\frac{M^{2}}{a^{2}} e^{-4 k a j}\left[\left(h_{\mu \nu}^{j+1}-h_{\mu \nu}^{j}\right)^{2}+\left(h_{\mu \nu}^{j+1}-h_{\mu \nu}^{j}\right) \square \phi^{j}+\left(\square \phi^{j}\right)^{3}\right] .
$$

The warp factor in front of the kinetic term tells us that the Planck scale on a site is

$$
M_{j}=M e^{-k a j}
$$

This is the warping we expect from the continuum. For the KK modes, we saw that to a good approximation $1 /(k a)$ modes have support at each site so we expect this parameter to play the role of $N$ in the previous section. So the effective Planck scale for the modes will be

$$
M_{n}=\frac{1}{\sqrt{k a}} M_{j_{n}}
$$

in agreement with the observation that $1 /(k a)$ in the warped case should play the role of $N$.

In fact, because we are approximating the KK modes for warped space by mapping to a position-dependent set of flat space solutions, we can simply use the flat space results if we just complete the map. Looking at the Lagrangian, in the approximation of Section 3.1, we see that the warped space Lagrangian for the $1 /(k a)$ modes around mode $n$ is equivalent to a flat space lattice with

$$
M_{\text {flat }} \rightarrow M_{j_{n}}=M e^{-k a j_{n}} \quad \frac{1}{a_{\text {flat }}} \rightarrow \frac{1}{a} e^{-k a j_{n}} \quad N_{\text {flat }} \rightarrow \frac{1}{k a} .
$$

Thus the strong coupling scale becomes

$$
\Lambda_{\text {flat }}=\left(\frac{M_{\text {flat }}}{N_{\text {flat }}^{5 / 2} a_{\text {flat }}^{4}}\right)^{1 / 5} \rightarrow \Lambda_{\text {warp }}=\left((k a)^{5 / 2} M_{j_{n}} \frac{1}{a^{4}} e^{-4 k a j_{n}}\right)^{1 / 5}=\left(\frac{1}{k a} M_{n} m_{n}^{4}\right)^{1 / 5},
$$


where $m_{n}=k e^{-k a j_{n}}$ is the mass of the lowest mode in the expansion around site $j$.

This result is superficially similar to that of flat space. The strong coupling scale is a factor of $N_{\text {flat }} \sim 1 /(k a)$ above the strong coupling scale for a single massive graviton. But there is a huge difference - the strong coupling scale does not depend on the size of the space we are latticizing. There is no dependence on the total number of lattice sites $N$, or equivalently on the IR scale $R=a e^{-k a N}$. In flat space, the strong coupling was determined by the lightest mode, but in warped space, it is determined by the lightest mode with support on the site $j$. For a $j$ close to the UV brane, all the modes which live there are much heavier than the $1 / R$ mode which would dominate if the space were flat.

However this is not the whole story. In warped space, there is not a single strong coupling scale; the strong coupling scale depends on the observer. In fact, there is an important difference between the scale associated with a particular mode and the scale that an observer on a particular site would see. For a mode of mass $m_{n}$, the strong coupling scale is the usual

$$
\Lambda_{\text {mode }} \sim\left(M_{n} m_{n}^{4}\right)^{1 / 5}
$$

This scale is heavier than $m_{n}$, so the mode is weakly coupled at energies near its mass. However, an observer at site $j$ would see $1 /(k a)$ modes. In particular, the mode of mass $m_{n}$ would be relevant even at energies as high as $m_{n} /(k a)$. When looking at the strong coupling scale on a site we must use $\Lambda_{\text {warp }}$ of Equation (54). The relevant Planck scale for the observer is $M_{j_{n}}=\sqrt{k a} M_{n}$. For example, with a lattice spacing $1 / a \sim M$ we would find that

$$
\Lambda_{\text {site }}=\Lambda_{\text {warp }}=M_{j_{n}} \sqrt{\frac{k}{M}}<M_{j_{n}} .
$$

So, if we only talk about modes, there is no strong-coupling problem in warped space. But observers localized at some position in the bulk (that is, on a particular lattice site) must encounter strong coupling before the local Planck scale. In asking about the lattice theory, it makes sense to consider the site basis, since otherwise the cut-off is determined solely by the IR regime.

We can understand these results in continuum language as well. In the warped case,

$$
\mathcal{L}=e^{-4 k a j} \frac{M_{5}^{3}}{a^{2}}\left(\Delta h_{\mu \nu}^{j}+\square \phi_{j}\right)^{2} \rightarrow M_{5}^{3} e^{-4 k y}\left(\partial_{y} h\right)^{2}+\frac{M_{5}^{3}}{a} e^{-4 k y}(\square \phi)\left(\partial_{y} h\right) .
$$

When we integrate by parts, the $\partial_{y}$ term hits the warp factor, so there are two pieces

$$
\frac{1}{a} e^{-4 k y}\left(\partial_{y} \phi-4 k \phi\right)(\square h)
$$

Initially, we might expect that for the long wavelength modes, with $\partial_{y} \sim 1 / R$, the $k \phi$ piece will dominate and prevent nonlocal effects. However, this would work only 
if there were modes spread out over the whole space. In warped space, there are not really any $1 / R$ modes. The wavelengths in the extra dimension are in fact limited by $k<\partial_{y}<1 / a$ - at each site, there are only $1 /(k a)$ modes. So $\partial_{y} \geq k$ and we can basically ignore the $k \phi$ term in (58).

Using $\hat{h}=\exp (-k y) h$, and adding the $a /(k a)=1 / k$ volume factor, the Lagrangian becomes

$$
\mathcal{L} \supset \frac{M_{5}^{3}}{k} \hat{h} \square \hat{h}+\frac{M_{5}^{3}}{k} \frac{1}{a} e^{-3 k y} \hat{h} \square\left(\partial_{y} \phi\right)+\frac{M_{5}^{3}}{k} \frac{1}{a^{2}} e^{-4 k y}(\square \phi)^{3} .
$$

The canonical propagating field is then

$$
\psi=\partial_{y} \phi^{c}=\frac{M_{5}^{3 / 2}}{k^{1 / 2} a} e^{-3 k y} \partial_{y} \phi,
$$

and the cubic scalar coupling becomes

$$
\frac{M_{5}^{3}}{k a^{2}} e^{-4 k y}(\square \phi)^{3} \rightarrow \frac{a k^{1 / 2}}{M_{5}^{3 / 2} \partial_{y}^{3}} e^{5 k y}\left(\square \phi^{c}\right)^{3} .
$$

For a mode of frequency $\partial_{y} \sim \omega$ this gives a strong coupling scale of

$$
\Lambda^{5}=\frac{M_{5}^{3 / 2} \omega^{3}}{a k^{1 / 2}} e^{-5 k y} .
$$

If we look at the individual modes, then $\omega \sim 1 / a(\operatorname{cf~Eq~(37))}$, and so the mode scale is

$$
\Lambda_{\text {mode }}^{5}=\frac{M_{5}^{3 / 2}}{\sqrt{k}} \frac{1}{a^{4}} e^{-5 k y},
$$

which is the same as $M_{n} m_{n}^{4}$ that we derived above. An observer at position $y$ is sensitive to wavelengths as high as $\omega \sim k$, which gives

$$
\Lambda_{\text {site }}=\left(M_{5}^{3} k^{5} a^{-2}\right)^{1 / 10} e^{-k y} .
$$

This is the same as the flat space interaction scale $\Lambda_{\text {flat }}=\left(M_{5}^{3} R^{-5} a^{-2}\right)^{1 / 10}$ with $k=R^{-1}$ the playing the role of the IR cutoff for the warped space.

\section{Conclusion}

We have shown that a straightforward discretization of the warped AdS geometry produces a low-energy theory which is valid above the scale of local curvature at any site. This is in fact sufficient for investigating many physical features, such as the renormalization group behavior of the theory as in [15-18]. However, the discretization does not allow us to reach the UV cutoff of the continuum theory. In 
the energy regime between the warped AdS scale and the warped higher-dimensional Planck scale, the theory acts like flat space and the same strong coupling problems as in flat space appear. This is not surprising; at energies above the local curvature, the theory approaches flat space, and therefore we do not expect manifestly holographic behavior. But it is extremely interesting that some of the holographic behavior of warped space is also manifested on the lattice; for example, we have found that the mass eigenvalues are globally geometrically spaced, with only $M / k$ modes localized near any particular site.

An important distinction from the flat space theory is that we can take the large volume limit because the UV and IR cutoffs are independent. In flat space, the UV cutoff goes down as the size of the space, $R$, is increased. In curved space, the UV cutoff only depends on the curvature scale, $k$; the cutoff in a particular region is completely ignorant of the total volume, or equivalently, the total number of sites. This cutoff is still below the local Planck scale, so even in the warped case, the lattice cannot be used as a regulator - divergences must still be cutoff by hand, or new physics must enter at a scale below the lattice spacing.

As in any non-renormalizable theory with a dimensionful scale, there is a natural limit to the lattice spacing, and thus a natural limit to the number of sites on the lattice. In warped space, this is a particularly strong bound. For example, in RS1 [1], the dimensionful scale is $M_{P}$, but the size of the space is set by $k e^{-k R}=T e V$ and so $N<30$. Nonetheless, on a larger warped space, approaching RS2 [2], 1/N effects can be parametrically ignored. Note that it is precisely because we can take the large volume limit with fixed lattice spacing that large $N$ is interesting. In flat space, because the UV cutoff decreases as the volume grows, in the large volume limit the cutoff goes to zero. In warped space, it remains above the local curvature scale.

Because the discretization of RS is sufficiently well behaved, we expect a similar discrete theory may be a useful tool for studying other geometries that exhibit holographic behavior. For example, the metric for de Sitter space and some black holes can be written in a warp-factor notation [14]. Thus their discretizations should be similar and may help unravel their holographic features. Another interesting example is local localization [19], in which the warp factor decreases to a minimum and then grows again. We expect that sites in the turnaround regime will have a low cutoff. However, modes localized on these sites will have very little support in the region where four-dimensional gravity applies. There is no reason to expect the massive mode of locally localized gravity that plays the role of the massive graviton to cause problems. Presumably locally localized gravity is far more general. The discrete version of theories with non-monotonic warp factor could be a useful tool for studying different examples of locally localized gravity, even with more than one extra dimension. 


\section{Acknowledgements}

We would like to thank J. Gallicchio and I. Yavin for discussing their results [20]. We would also like to thank Y. Shadmi and R. Mahbubani for discussions. After this work was published, we were informed that a related discussion can be found in [21]. The work of LR was supported in part by NSF Award PHY-0201124.

\section{References}

[1] L. Randall and R. Sundrum, Phys. Rev. Lett. 83, 3370 (1999) arXiv:hep-ph/9905221.

[2] L. Randall and R. Sundrum, Phys. Rev. Lett. 83, 4690 (1999) arXiv:hep-th/9906064.

[3] N. Arkani-Hamed, A. G. Cohen and H. Georgi, Phys. Rev. Lett. 86, 4757 (2001) arXiv:hep-th/0104005.

[4] N. Arkani-Hamed, H. Georgi and M. D. Schwartz, Annals Phys. 305, 96 (2003) arXiv:hep-th/0210184.

[5] N. Arkani-Hamed and M. D. Schwartz, Phys. Rev. D 69, 104001 (2004) arXiv:hep-th/0302110.

[6] M. D. Schwartz, Phys. Rev. D 68, 024029 (2003) arXiv:hep-th/0303114.

[7] L. Randall, Y. Shadmi and N. Weiner, JHEP 0301, 055 (2003) arXiv:hep-th/0208120.

[8] H. Abe, T. Kobayashi, N. Maru and K. Yoshioka, Phys. Rev. D 67, 045019 (2003) arXiv:hep-ph/0205344.

[9] A. Falkowski and H. D. Kim, JHEP 0208, 052 (2002) arXiv:hep-ph/0208058.

[10] A. Katz and Y. Shadmi, JHEP 0411, 060 (2004) arXiv:hep-th/0409223.

[11] T. Bhattacharya, C. Csaki, M. R. Martin, Y. Shirman and J. Terning, arXiv:hep-lat/0503011.

[12] O. Andreev and W. Siegel, Phys. Rev. D 71, 086001 (2005) arXiv:hep-th/0410131.

[13] S. R. Coleman, J. Wess and B. Zumino, Phys. Rev. 177, 2239 (1969). C. G. . Callan, S. R. Coleman, J. Wess and B. Zumino, Phys. Rev. 177, 2247 (1969). 
[14] L. Randall, V. Sanz and M. D. Schwartz, JHEP 0206, 008 (2002) arXiv:hep-th/0204038.

[15] A. Pomarol, Phys. Lett. B 486, 153 (2000) arXiv:hep-ph/9911294.

[16] L. Randall and M. D. Schwartz, Phys. Rev. Lett. 88, 081801 (2002) arXiv:hep-th/0108115.

[17] L. Randall and M. D. Schwartz, JHEP 0111, $003 \quad$ (2001) arXiv:hep-th/0108114.

[18] W. D. Goldberger and I. Z. Rothstein, Phys. Rev. Lett. 89, 131601 (2002) arXiv:hep-th/0204160.

[19] A. Karch and L. Randall, Phys. Rev. Lett. 87, 061601 (2001) arXiv:hep-th/0105108.

[20] J. Gallicchio and I. Yavin, arXiv:hep-th/0507105.

[21] G. Cognola, E. Elizalde, S. Nojiri, S. D. Odintsov and S. Zerbini, Mod. Phys. Lett. A 19, 1435 (2004) arXiv:hep-th/0312269. 